\section{La dieta sin gluten y los alimentos libres de gluten}

\author{
Gluten-free diet and \\ gluten-free foods
}

\begin{abstract}
The gluten-free foods global market has significantly grown during the last decade. Gluten-free diet represents the treatment for celiac disease, non-celiac gluten sensitivity and wheat allergy. Another group of persons that follows gluten-free diet has emerged, perceiving this diet as healthier feeding habit. It is especially this latter group that has increased to such an extent that they modified the market of gluten-free foods, improving availability of these products. However, because their demands and requirements are different, gluten content control of many of the currently existing local gluten-free foods is unsatisfactory. We present the information available on these issues, emphasizing the few local data available, which refer to school-age snacks and the "Basic Food Basket", with and without gluten. Local studies show that gluten-free snacks for school-age children and the gluten-free Basic Food Basket are less available, their cost is higher and less than $8 \%$ meet FAO/WHO nutritional recommendations.

Key words: gluten, gluten-free diet, celiac disease, gluten-free food, gluten-free food market.
\end{abstract}

\section{INTRODUCCIÓN}

El mercado mundial de los productos sin gluten ha tenido un aumento sin precedente en los últimos años (1). Entre las razones que explican este aumento está el aumento significativo que han tenido los problemas de salud relacionados con la ingesta de gluten (enfermedad celíaca) $(E C)(2,3)$, sensibilidad no celíaca al gluten (SNCG) (4-7), y alergia al trigo (AT) $(6,8$, 9), además de un grupo creciente de personas aparentemente sanas que optan por una dieta sin gluten por considerarla más saludable. Revisaremos en qué consisten cada uno de estos cuadros y su relación con el gluten de la dieta, y comentaremos los alimentos sin gluten disponibles a nivel nacional.

\section{LAS PATOLOGÍAS QUE REQUIEREN TRATAMIENTO CON DLG}

Enfermedad celíaca. Condición crónica autoinmune que resulta de la interacción de genes que aportan el riesgo, el gluten de la dieta (ambiente), el sistema inmune que actúa como efector, y la microbiota intestinal, cuyo rol aún está en estudio. En Chile la frecuencia de EC se estima en alrededor de 0,6-0,8\% de la población (10); tiene manifestaciones clínicas muy variables, que incluyen síntomas digestivos y extra digestivos. Esta condición es la más estudiada y la que más aporta al
Virginia Estévez Magdalena Araya

Laboratorio de Gastroenterología, Unidad de Nutrición Humana, Instituto de Nutricion y Tecnologia de los Alimentos (INTA), Universidad de Chile, Santiago, Chile.

Dirigir la correspondencia a: Profesora Magdalena Araya INTA, Universidad de Chile El Líbano 5524 Macul - Santiago - Chile Fono: 56229781411 Email: maraya@inta.uchile.cl

Este trabajo fue recibido el 22 de Julio de 2016 y aceptado para ser publicado el 12 de Octubre de 2016.

conocimiento de lo que ocurre en el intestino delgado cuando se ingiere gluten. Su único tratamiento eficaz es la dieta libre de gluten (DLG) $(3,11)$, mantenida de manera estricta y por toda la vida (tabla 1). Aunque despiertan gran interés entre los celíacos, hasta ahora no hay opciones de tratamientos no dietarios (12). Por otro lado y salvo excepciones (casos refractarios), tampoco hay terapia medicamentosa.

Sensibilidad no celíaca al gluten. Aunque reconocida desde hace décadas (13), la descripción moderna tiene menos de cinco años $(4,6)$. De acuerdo a los consensos vigentes entre especialistas, se caracteriza por la aparición rápida de síntomas tras ingerir trigo/gluten, que pueden ser digestivos y/o extra digestivos. En los pacientes se demuestra ausencia de fenómenos alérgicos y autoinmunes, los auto anticuerpos medidos en sangre son negativos y las biopsias yeyunales son normales (lo cual de acuerdo al conocimiento actual descarta la EC) $(4,6$, ). Sin embargo, hay clara mejoría clínica en respuesta a la DLG, con desaparición de la sintomatología y rápida reaparición de ellos al realizar una contraprueba con gluten $(4,6)$. Se discute si se trata de una respuesta al gluten y/o a otras proteínas del trigo (14). Hasta ahora, el tratamiento consiste en eliminar o disminuir la ingesta de trigo hasta la desaparición de los síntomas (tabla 1). Tanto en Chile como 
globalmente, la información disponible no permite aún estimar su prevalencia ni su curso clínico.

Alergia al trigo. Se trata de una reacción adversa mediada inmunológicamente que forma parte de las alergias alimentarias. Hasta hace pocos años recibía escaso interés porque era infrecuente; hoy está bien sustentada en la literatura internacional cuando el mecanismo subyacente es mediante IgE, aunque también se ha describe que puede ser causa de gastroenteropatía eosinofílica (15). En esta condición hay ausencia de fenómenos de autoinmunidad y también se discute si se debe al gluten u otras proteínas contenidas en el grano de trigo. Se induce cuando el trigo es ingerido, tiene contacto con la piel o se aspira por la vía respiratoria, y los síntomas pueden aparecer en cualquiera de estos sistemas. El tratamiento consiste en una dieta estricta de eliminación de alimentos que contengan trigo (15) (tabla 1). En Chile no hay información al respecto, pero en opinión de los especialistas hasta ahora sería infrecuente.

Es relevante considerar que las manifestaciones clínicas de la EC, sensibilidad no celíaca al gluten y alergia al trigo son inespecíficas, se sobreponen marcadamente entre ellas (tabla 2) y además, con la sintomatología de otros cuadros como el síndrome de intestino irritable, o con el derivado de la alta ingesta de FODMAPs ("Fermentable Oligo- Di- Monosaccharides and Polyols") (16), carbohidratos de cadena corta, de tamaño molecular relativamente pequeño, que son pobremente absorbidos, ejercen efecto osmótico y constituyen sustrato para la microbiota (17). Su metabolización por ésta despierta la sintomatología típica con distensión abdominal, meteorismo y dolor abdominal $(17,18)$. Actualmente, el tratamiento de quienes sufren síntomas derivados de la ingesta de FODMAPs consiste en eliminar o disminuir los alimentos que los contengan hasta el punto que desaparezca la sintomatología (16). Se desconoce su prevalencia y las consecuencias que pueda tener a largo plazo la mantención de una dieta alta en estos compuestos. Es necesario tener en cuenta que todos los cuadros mencionados pueden tener manifestaciones clínicas similares.

"Vivir sin gluten" como opción de alimentación saludable

En la actualidad, un grupo considerable y creciente de personas sin diagnóstico que lo justifique están optando por la DLG, porque la consideran una opción de alimentación más saludable. El creciente interés de la población chilena en el gluten y la DLG se ve reflejado en las más de 33.000 búsquedas promedio mensuales relacionadas a "gluten" en Google (19). De hecho, en 2015 "gluten" fue la cuarta palabra más buscada en google en el país dentro de la categoría "Qué". Actualmente, no hay información acerca de hasta qué punto la DLG es estricta cuando se trata de grupos que la siguen como opción saludable; tampoco se sabe si tiene efectos adversos a largo plazo, ya que es sabido que los ASG frecuentemente contienen mayor cantidad de grasas y/o carbohidratos y son pobres en vitaminas y minerales, porque no son fortificados (20). Un punto favorable es que este grupo de población ha

\section{TABLA 1}

Restricción del gluten de la dieta que deben respetar las personas con sensibilidad no celíaca al gluten, enfermedad celíaca, alergia al trigo o "dieta saludable libre de gluten"

Condición

Sensibilidad no celíaca al gluten

Enfermedad celíaca

Alergia al trigo

Moda - dieta saludable sin gluten
Gluten contenido en la dieta.

Disminución del consumo hasta la desaparición de la sintomatología.

Consumo por debajo del punto de corte (Chile= $3 \mathrm{ppm}$ ).

Eliminación total, incluso trazas de gluten. Sin punto de corte.

Disminución del consumo que la persona acepta como saludable.

\section{TABLA 2}

Cuadros clínicos de diversas etiologías y su relación con la ingesta de gluten en la dieta.

Relación a la dieta

Relacionadas al gluten

No relacionadas al gluten
Mecanismo principal

Autoinmunidad

Inmunidad

No inmune

Defectos enzimáticos digestivos

Farmacológicas

Otros
Condición

Enfermedad celíaca

Alergia al trigo

Sensibilidad no celíaca al gluten

Intolerancia lactosa, Intolerancia sacarosa-isomaltosa Intolerancia trehalosa

Jarabes, comprimidos FODMAPS*

$\mathrm{SII**}$

\footnotetext{
* = carbohidratos fermentables de cadena corta.
}

${ }^{* *}=$ síndrome de intestino irritable. 
conseguido mover el mercado de ASG, estimulando a la industria de alimentos a desarrollar o importar nuevos productos, lo que ha favorecido la disponibilidad de alimentos para pacientes que necesitan adherir a la DLG como tratamiento. Sin embargo, este grupo también compite por la compra de los ASG más baratos, y por otro lado, como no tienen la rigurosidad de exigir alimentos demostradamente seguros, favorecen la producción de alimentos que se expenden sin control alguno. Un buen ejemplo de esto último se evidencia en la creciente oferta de menús y preparaciones listas para el consumo sin gluten que se ofrecen hoy día en restaurantes, cafeterías e incluso en páginas web y redes sociales, sin control del contenido de gluten, ni de las materias primas que usan para sus preparaciones, ni de la aplicación de Buenas Prácticas de Manufactura, todos ellos exigibles para que el alimento o producto sea seguro para el consumo d los celíacos.

\section{La dieta sin gluten, los consumidores} y el mercado de ASG

Los ASG despiertan interés porque a pesar que el conocimiento de la EC ha aumentado sustancialmente en las últimas décadas, hasta hoy día el único tratamiento eficaz de esta enfermedad es la DLG $(6,11)$. Lo mismo ocurre con los cuadros de SNCG y AT, que igualmente deben eliminar el gluten de la dieta $(4,6)$. El aumento de la prevalencia de estas patologías ciertamente obedece a múltiples factores, entre los destacan el acceso a nuevas herramientas diagnósticas, mayor conciencia sobre estos cuadros por parte de la población y de los profesionales que deben buscarlas y diagnosticarlas, y un aumento de la prevalencia real de los cuadros asociados a la ingesta de gluten (2). Es curioso que el segmento de población que más ha influido en el mercado de ASG sea este grupo creciente de personas que opta por una alimentación sin gluten, a pesar de no tener un diagnóstico que la obligue a ello.

Muchos se preguntan a qué se debe el alza significativa de los cuadros asociados a la ingesta de gluten. Una de las hipótesis actuales más interesantes que intenta explicar el fenómeno invoca el aumento reciente de $\sim 3$ veces de la ingesta de gluten exógeno, o sea, aquel agregado con fines tecnológicos/industriales para mejorar las características de emulsificación, cohesividad, viscoelasticidad, gelificación, espuma, entre otros, en los productos procesados $(21,22)$. Se calcula que en 1977 la ingesta de gluten era 136 g/persona/ año, mientras que en 2015 sería de 408 g/persona/año (22). Parte del apoyo a la hipótesis es que el aumento de la ingesta de gluten coincide con el marco de tiempo en que se ha observado el aumento de frecuencia de la EC y la aparición de los otros cuadros relacionados a la ingesta de gluten (22, 23). Si aceptamos esta hipótesis, en el caso de Chile y basados solamente en el dato de consumo de 96 kilogramos de pan per cápita al año (24), se puede estimar que la ingesta de gluten sería de 700 g o más por persona/año, dependiendo de la calidad del trigo analizado.

La situación histórica en todo el mundo (y especialmente en (hile) ha sido que la disponibilidad de ASG seguros era muy limitada y que mantener una DLG estricta resultaba de enorme dificultad. El aumento del número de personas que consumen dietas sin gluten ha llevado a que el mercado de ASG crezca y hoy, de acuerdo a estudios realizados por la consultora Marketsandmarkets, especialista en este tipo de análisis de fluctuación de mercado en E.E.U.U., el mercado de los ASG alcanzó los 4.63 billones de dólares anuales en 2015 y llegará a 7.59 en 2020 (25). Actualmente, en la mayoría de los segmentos de la población hay confusión acerca de qué significa una dieta sin gluten, que son y cómo se reconocen los ASG y cómo es el mercado de ASG en Chile. Varios factores favorecen esta situación, los cambios en hábitos de alimentación han ocurrido rápidamente, el mercado de productos sin gluten ha crecido en corto tiempo, las nuevas tecnologías de alimentos facilitan el desarrollo de ASG cada vez más elaborados y gratos para los consumidores, el grupo de población general que opta por consumir ASG crece aceleradamente y presenta necesidades y expectativas distintas, y el Estado reacciona lentamente en mejorar las normativas pertinentes y su fiscalización.

\section{El gluten como ingrediente, gluten oculto y contaminación cruzada.}

El gluten se refiere a un grupo de proteínas ricas en prolaminas, presentes en el trigo (gluten), cebada (hordeinas), y centeno (secalinas) (21). Es ampliamente utilizado por la industria de alimentos debido a sus características físicoquímicas, que inducen mejoras sustanciales en los productos que lo contienen. Su visco-elasticidad es fundamental para la producción de panes y productos horneados, mejorando la textura y la forma, la retención de agua y grasas y la extensibilidad de las masas, formando emulsiones, espumas y geles y sirviendo de vehículo para aromatizantes y colorantes. Además, disminuye los costos de producción al ser usado como sustituto de proteínas animales o "fillers" (21). Todo esto implica que si es incorporado al producto dentro de algún tipo de aditivo, no aparece declarado como tal entre los ingredientes.

El contenido de gluten en los alimentos varía dependiendo de los elementos que lo componen, y la formulación y procesamiento que sufren durante su elaboración. En la dieta "libre de gluten" el contenido de gluten no es cero. Esta dieta se basa en general en una combinación de: i) alimentos naturalmente libres de gluten o mínimamente procesados y no contaminados (como carne fresca, pescado, leche, huevos, frutas, verduras) y ii) alimentos procesados en que el gluten es sustituido por otros componentes, como cereales y almidones sin gluten (pan de maíz, galletas de arroz, entre otros), los que se procesan manteniendo buenas prácticas de manufactura que impiden su contaminación (21). De esta manera, la cantidad de gluten en el producto final queda por debajo de un "punto de corte" (ver más adelante).

Un alimento procesado puede contener gluten: 1) como ingrediente: cuando se utiliza en su preparación trigo, cebada, centeno o sus derivados; 2) como gluten oculto: cuando al producto se adiciona cualquier aditivo que contenga gluten, que habitualmente no es declarado; 3) Contaminación cruzada: alude a la introducción involuntaria de gluten en un alimento naturalmente carente de él o procesado como "libre de gluten". Puede ocurrir: a) durante el proceso de elaboración del alimento al compartir líneas de producción, equipos, o espacios en los que se procesan alimentos con gluten, o b) en la casa o servicio de alimentación, al exponer el alimento o preparación a utensilios o espacios contaminados. La presencia de gluten oculto y la contaminación cruzada explican por qué hoy día no basta leer el listado de ingredientes del producto para decidir si un producto es seguro para consumo de los celíacos. Es necesaria la aplicación de Buenas Prácticas de manufactura (BPM) y la medición de prolaminas en el producto final listo para ser consumido.

\section{El punto de corte, el Codex, la situación en otros países y en Chile}

Desde el punto de vista teórico, la mayoría de los autores están de acuerdo en que para el paciente celíaco "a menos 
gluten mejor". Sin embargo, decidir cuánto gluten puede consumir el celíaco sin sufrir daño ha sido y es tema de arduo debate. Dada la dificultad de eliminar completamente el gluten de los alimentos, el consenso internacional maneja un "punto de corte", que define cuánto gluten puede contener realmente un "alimento libre de gluten". El Codex Alimentarius (26) es el organismo internacional que, sin tener fuerza de ley, da la recomendación general. En su última reunión (2012) fijó el punto de corte en 20 ppm (o partes por millón o miligramos de gluten por kilogramo de producto). Algunos países han decidido que la evidencia científica actual disponible sugiere que este valor debiera ser menor y voluntariamente han bajado el punto de corte para su país a $<10$ ppm o <3ppm. Aunque variados grupos promueven los valores de punto el corte más altos, la FDA (Food and Drug Admnistration, EEUU) en su análisis de Evaluación de Riesgo, en 2011 calculó un punto de corte seguro para proteger de una respuesta clínica y otro para la respuesta histológica a todos los celíacos, resultando ambos menores de 1ppm (27).

En Chile, el Reglamento Sanitario de los Alimentos (28) fija los requerimientos que deben cumplir los productos que portan un rótulo "libre de gluten" (o símbolo de la espiga tachada), pero es escasamente fiscalizada. Establece que: i) la empresa que lo produce debe contar con un "Programa de Buenas Prácticas de Manufactura" que asegure la no contaminación cruzada en la cadena de producción, ii) el producto debe contar con una medición de prolaminas realizado en el producto terminado, listo para el consumo, menor que el punto de corte chileno (3 ppm, Decreto 134/14, 2015), y iii) las harinas y el pan deben contener las vitaminas y minerales establecidas en el artículo 350 del mismo Reglamento, para panes y harinas para uso de panificación.

\section{El mercado de alimentos sin gluten en Chile Experiencias nacionales}

La información local acerca del mercado de ASG es muy escasa. La adherencia a la DLG se estima difícil debido al alto consumo de pan en el país (24). La alta prevalencia de sobrepeso/obesidad (29) ha llevado a implementar numerosas campañas (30-31) tendientes a fomentar hábitos de alimentación saludables, lo que pueden haber favorecido la decisión de adoptar dietas "libre de", incluyendo la dieta que no contenga gluten. Al no estar caracterizado, este grupo de personas que consume productos sin gluten sin tener un diagnóstico que se los exija ha hecho más difícil estimar la cuantía y las características de los cambios que han sufrido los productos sin gluten disponibles en el mercado, ya que la estimación se hacía históricamente en base al consumo que podrían tener los pacientes celíacos. Recientemente se han realizado dos estudios acerca de la disponibilidad, costo y calidad nutricional de los ASG en la Región Metropolitana. En el primero (32) se evaluaron productos frecuentemente consumidos como colaciones escolares. Siguiendo metodologías previamente descritas (33-34), se eligieron zonas representativas de los niveles socioeconómicos alto, medio y bajo, registrando las "colaciones" con y sin gluten que se ofrecían en los 5 tipos de locales de venta que exige y define el diseño utilizado (33-34): supermercados grandes de alta calidad, intermedios, de distribución, tiendas naturistas y "pequeñas tiendas de la esquina". Se registró información acerca de los productos en que se encontró al menos un equivalente sin gluten. Estos resultaron ser lácteos (cajas de leches y yogures), cereales (en caja y en barra) y productos horneados (diversos tipos de galletas y queques). Se determinó la disponibilidad, costo y calidad nutricional (calorías totales, grasas y azúcares totales y sodio), comparando siempre los productos con y sin gluten. De los 1562 productos registrados en que hubo un equivalente sin gluten, los etiquetados "sin gluten" tuvieron menor disponibilidad, con diferencias estadísticamente significativas entre el nivel socio económico alto y bajo (chi cuadrado, $\mathrm{p}<0.05)$, y sus precios fueron más altos. A excepción de las calorías totales y el sodio de los cereales en el nivel socio económico alto, menos de un tercio de los productos sin gluten cumplen las recomendaciones nutricionales de FAO/ OMS. El resultado más relevante fue que solo $7,8 \%, 7,3 \%$ y $7,3 \%$ de los productos sin gluten tenían el total de calorías, grasas, azúcares totales y sodio dentro de las recomendaciones nutricionales, en los niveles socioeconómicos alto, medio y bajo, respectivamente (32).

En un segundo estudio evaluamos la Canasta Básica Familiar Sin Gluten (CBF/SG) (35). El concepto CBF es usado en nuestro país para establecer el consumo mínimo de alimentos que deben mantener cada integrante de una familia que en promedio está formada por 4 miembros, para cubrir los requerimientos nutricionales. Se determinó la disponibilidad, costo, principales ingredientes y calidad nutricional de la CBF/SG, comparándola con la CBF habitual. Los datos se recolectaron en una de las 5 comunas de alta prioridad social del Ministerio de Desarrollo Social. Se buscaron los productos que constituían la CBF en los mismos 5 tipos de locales descritos en el estudio anterior (32) y se les buscó su equivalente sin gluten. No se encontraron supermercados que cumplían la definición de grandes/de alta calidad en el área evaluada y se identificaron "pares" sin gluten solo en el supermercado de tamaño intermedio y de distribución. De los 53 alimentos que conforman la CBF, se evaluaron los 34 que contienen gluten o existían antecedentes de estar contaminados. Solo en 19 de los 34 (55,8\%) se encontró un equivalente sin gluten, los que conformaron la CBF/SG. La disponibilidad de la CBF/SG fue $42 \%$ la de la CBF habitual (36). El costo de los 19 alimentos en que se pudo hacer la comparación en un equivalente sin gluten y de bajo precio, fue $\$ 101.000 /$ mes para los sin gluten versus $\$ 43.000 /$ mes para los con gluten. Un estudio anterior, con metodología no enteramente comparable, en 2009 ya había descrito que la CBF/SG era más costosa que la CBF habitual (37). En nuestro estudio algunos "panes y horneados", que fue la categoría que mostró las mayores diferencias, se observaron precios hasta 529\% mayor. Asimismo, la CBF/SG en el grupo de panes y cereales tuvo menor contenido proteico hasta $69 \%$, y los ingredientes principales se limitaron a harina de arroz, maíz y almidón de mandioca, sin ninguna fortificación. Los resultados de los estudios descritos sugieren que tanto los escolares como en general las personas que dependen de la CBF/SG tienen un problema significativo de disponibilidad y mayor costo de alimentación. El menor contenido proteico los pone en claro riesgo nutricional, lo que es especialmente relevante en los niños que tienen una mayor tasa de crecimiento. La baja disponibilidad de la CBF/SG claramente dificulta la adherencia a la DLG y favorece las complicaciones, aumentando el costo económico para la familia y el sistema de salud que la atiende. Y muy importante, claramente afecta la calidad de vida de los afectados. En resumen, el análisis de la situación chilena actual indica que los actores involucrados en el mercado de ASG deben tomar las responsabilidades que les corresponde, es decir, los individuos que requieren ASG deben exigir productos seguros, el Estado debe mejorar las normativas y fiscalizar su aplicación, y las empresas deben comprometerse 
con los conceptos de la Responsabilidad Social Empresarial en la producción de ASG, ampliando la gama de productos seguros y mejorando su calidad nutricional, especialmente proteica y de fortificación.

\section{RESUMEN}

El mercado global de los productos sin gluten ha tenido un aumento sin precedente en la última década. La dieta sin gluten constituye el tratamiento de la enfermedad celíaca, la sensibilidad no-celíaca al gluten y de la alergia al trigo. Recientemente ha aparecido otro grupo de personas que elimina el gluten por considerarlo una opción de alimentación más saludable. Es especialmente este último grupo que ha logrado mejorar el mercado de alimentos sin gluten, pero sus requerimientos son distintos, por lo que parte del aumento del mercado ha sido en base de productos sin control adecuado de su contenido de gluten. Analizamos la información existente, poniendo énfasis en los datos nacionales recientemente obtenidos acerca de las colaciones escolares y la Canasta Básica Familiar sin gluten. En ambos casos la disponibilidad de productos equivalentes sin gluten es menor, el costo tres o más veces mayor y menos del $8 \%$ de ellos cumplen las recomendaciones nutricionales de la FAO/OMS.

Palabras clave: gluten, dieta libre de gluten, enfermedad celíaca, alimentos sin gluten, mercado alimentos sin gluten.

Agradecimientos: Este estudio fue financiado por el INTA, Universidad de Chile. La entidad financiera no tuvo influencia alguna en el diseño del estudio; en la recolección, análisis o interpretación de los datos; en la preparación, revisión o aprobación del manuscrito

Conflicto de interés: Los autores declaran no tener conflicto de interés.

\section{BIBLIOGRAFÍA}

1. Foschia, M., et al., Nutritional therapy - Facing the gap between coeliac disease and gluten-free food. Int J Food Microbiol, 2016.

2. Rubio-Tapia, A., et al., Increased prevalence and mortality in undiagnosed celiac disease. Gastroenterol, 2009. 137(1): 88-93.

3. Catassi, C., et al., Natural history of celiac disease autoimmunity in a USA cohort followed since 1974. Ann Med, 2010; 42(7): 530-8.

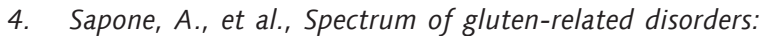
consensus on new nomenclature and classification. BMC Med, 2012; 10: 13.

5. Sapone, A., et al., Differential mucosal IL-17 expression in two gliadin-induced disorders: gluten sensitivity and the autoimmune enteropathy celiac disease. Int Arch Allergy Immunol, 2010; 152(1): 75-80.

6. Catassi, C., et al., Non-Celiac Gluten sensitivity: the new frontier of gluten related disorders. Nutrients 2013. 5(10): 3839-53.

7. Nijeboer, P., et al., Non-celiac gluten sensitivity. Is it in the gluten or the grain? J Gastrointest Liver Dis. 2013. 22(4): 435-40.

8. Siripipattanamongkol, N., et al., Age of Resolution from lgEMediated Wheat Allergy. Asian Pac J Allergy Immunol, 2016.

9. Winberg, A., et al., Assessment of Allergy to Milk, Egg, Cod, and Wheat in Swedish Schoolchildren: A Population Based Cohort Study. PLoS One, 2015. 10(7): p. e0131804.

10. Ministerio de Salud and Gobierno de Chile, "Segunda
Encuesta Nacional de Salud" (National Health Survey) 2009. Disponible en: http://www.minsal.cl/. [Consultado el 29 de junio 2016].

11. Lionetti $E$, Catassi C. New clues in celiac disease epidemiology, pathogenesis, clinical manifestations, and treatment. Intern Rev Immunol 2011; 30 (4): 219-31.

12. Plugis NM, Khosla C. Therapeutic approaches for celiac disease. Best Pract Res Clin Gastroenterol. 2015; 29 (3): 503-21.

13. Ellis $A$, Linaker $B$. Non-celiac gluten sensitivity? Lancet 1978; 1(8078):1358-9.

14. Nijeboer P, Bontkes $H$, Mulder C, Bouma G. Non-celiac gluten sensitivity. Is it in the gluten or the grain?. J Gastrointestin Liver Dis. 2013; 22 (4): 435-40.

15. Cianferoni $A$. Wheat allergy: diagnosis and management. J Asthma Allergy 2016;9: 13-25.

16. De Giorgio, R., U. Volta, and P.R. Gibson, Sensitivity to wheat, gluten and FODMAPs in IBS: facts or fiction? Gut. 2016. 65(1): 169-78.

17. Barrett, J. S., \& Gibson, P. R. (2012). Fermentable oligosaccharides, disaccharides, monosaccharides and polyols (FODMAPs) and nonallergic food intolerance: FODMAPs or food chemicals? Therapeutic Advances Gastroenterol, 5(4), 261-268. http://doi.org/10.1177/1756283X11436241

18. Barrett, J.S., et al., Dietary poorly absorbed, short-chain carbohydrates increase delivery of water and fermentable substrates to the proximal colon. Aliment Pharmacol Ther, 2010. 31(8): 874-82.

19. Adwords. Búsquedas en Google relacionadas con "gluten" 2015. Disponible en: https://adwords.google.es/keywordplanner. [Consultado el 29 de junio 2016].

20. do Nascimento AB, Fiates GM, Dos Anjos A, Teixeira E. Analysis of ingredient lists of commercially available gluten-free and gluten-containing food products using the text mining technique. Internat J Food Sci Nutr. 2013 Mar;64(2):217-22. PubMed PMID: 22946669.

21. Shewrya PR, Tathamc AS. Improving wheat to remove coeliac epitopes but retain functionality. J Cereal Sci. 2016; 67: 12-21.

22. Fardet $A$. Wheat-based foods and non-celiac gluten/wheat sensitivity: Is drastic processing the main key issue? Med Hypotheses. 2015; 85 (6): 934-9.

23. Kasarda DD. Can an increase in celiac disease be attributed to an increase in the gluten content of wheat as a consequence of wheat breeding? J Agric Food Chem. 2013; 61 (6): 1155-9.

24. Ministerio de Salud Pública (MINSAL). Encuesta Nacional de Consumo de Alimentos (ENCA) 2014 Santiago, Chile, 2015. Disponible en: http://web.minsal.cl/sites/default/ files/ENCA-INFORME_FINAL.pdf. [Consultado el 29 de junio 2016].

25. Markets and Markets. Gluten-Free Products Market by Type (Bakery Products, Pizzas \& Pastas, Cereals \& Snacks, Savories, and Others), Source (Oilseeds \& Pulses, Rice \& Corn, Dairy \& Meat Products, and Other (rops), \& by Region - Global Trends \& Forecast to 2020. Disponible en: http://www.marketsandmarkets.com/PressReleases/ gluten-free-products.asp. [Consultado el 29 de junio 2016].

26. Food and Agriculture Organization World Health Organization (FAO/WHO). Codex Committee on Nutrition and Foods for Special Dietary Uses. Codex standard for foods for special dietary use for persons intolerant to gluten. World Health Organization, Rome, Italy 2008.

27. Food and Drug Administration. Health Hazard Assessment 
for Gluten Exposure in Individuals with Celiac Disease: Determination of Tolerable Daily Intake Levels and Levels of Concern for Gluten. Office of Food Safety/Center of Food Safety and Applied Nutrition, Food and Drug Administration, USA, 2011.

28. Ministerio de Salud. Reglamento Sanitario de los Alimentos. Disponible en: http://www.sernac.cl/wp-content/ uploads/2012/11/reglamento-sanitario-alimentos-2011. pdf [Consultado el 29 de junio 2016].

29. Ministerio de Salud. Encuesta Nacional de Salud. Indicadores de obesidad en la población chilena 2010. Disponible en: http://www.minsal.gob.cl/portal/url/item/9ad9cbfb 71ca4705e04001011e010283.pdf [Consultado el 29 de junio 2016].

30. La Tercera. Campaña para luchar contra el sedentarismo. Disponible en: www.latercera.com/.../659-632394-9-lanzan-campana-para-luchar-contra-el-sedentarismo. [Consultado el 13 de junio 2016].

31. El Periscopio. Junaeb comenzó campaña contra la obesidad estudiantil. Disponible en: www.chilevision.cl/noticias/ chvnoticias/. [Consultado el 13 de junio 2016].
32. Oyarzún A, Nakash T, Ayala J, Lucero Y, Araya M (2015) Following Gluten Free Diet: less available, higher cost and poor nutritional profile of gluten-free school snacks. Internat J Celiac Dis; 3. doi:10.12691/ijcd-3-3-3

33. Singh, J. and K. Whelan, Limited availability and higher cost of gluten-free foods. J Hum Nutr Diet, 2011. 24(5): 479-86.

34. Kinsey, L., S.T. Burden, and E. Bannerman, A dietary survey to determine if patients with coeliac disease are meeting current healthy eating guidelines and how their diet compares to that of the British general population. Eur J Clin Nutr, 2008. 62(11): 1333-42.

35. Estévez V, Ayala J, Vespa C, Araya A. The gluten-free Basic Food Basket. A problem of availability, cost and nutritional composition. Eur J Clin Nutr, aceptado para publiación, 2016.

36. Observatorio Social del Ministerio de Desarrollo Social (2014). Costo de la canasta básica de alimentos noviembre 2014. Santiago, Chile.

37. Castillo C, Rivas C. Costo canasta básica de alimentos para celiacos en Chile. Rev Méd Chil. 2008. 136, 613-9. 